\title{
Actividades de apoyo de la gestión logística en la industria petrolera división occidente
}

\author{
Support activities for logistics management in the oil industry west division
}

\begin{tabular}{l|r}
\cline { 2 - 2 } & Danny Garcia \\
\hline $\begin{array}{l}\text { Artículo recibido en mayo 2020 } \\
\text { Arbitrado en junio 2020 } \\
\text { Publicado en septiembre 2020 }\end{array}$ & dmgm77@gmail.com \\
& ORCID: 0000-0001-6128-7605 \\
Industria petrolera, Venezuela
\end{tabular}

RESUMEN

Palabras clave: Este artículo pretende caracterizar las actividades de apoyo de la gestión logística en la industria petrolera División occidente, bajo una investigación descriptiva, con un diseño de campo no experimental y transeccional. La población quedó constituida por los gerentes y líderes pertenecientes a las gerencias de operaciones y mantenimiento a taladro, a quienes se les aplico un cuestionario con alternativas de frecuencia compuesto por 11 ítems, el cual fue validado por expertos. Para determinar la confiabilidad se aplicó el método de Alfa de Cronbach, cuyo valor fue de 0,98. La información fue procesada a través de la estadística descriptiva específicamente mediante la media aritmética. Los resultados permitieron concluir que existe alta presencia del almacenamiento, manejo de materiales, compras, cooperación con producción y mantenimiento de información como actividades de apoyo de la gestión logística.

Actividades de apoyo, almacenamiento, compras, cooperación con producción, gestión logística, manejo de materiales, mantenimiento de información

ABSTRACT

Keywords:

This article tries to characterize the activities of logistic management support in the oil industry Western Division, under a descriptive investigation, with a non-experimental and transectional field design. The population was made up of managers and leaders belonging to the operations and drilling management departments, to whom a questionnaire with frequency alternatives consisting of 11 items was applied, which was validated by experts. To determine reliability, the Cronbach's Alpha method was applied, whose value was 0.98 . The information was processed through descriptive statistics specifically using the arithmetic mean. The results allowed to conclude that there is a high presence of storage, material handling, purchasing, cooperation with production and maintenance of information as support activities for logistics management.

Support activities, warehousing, purchasing, cooperation with production, logistics management, material handling, information maintenance 


\section{INTRODUCCIÓN}

La nueva realidad competitiva presenta un campo de batalla en donde la flexibilidad, la velocidad de llegada al mercado y la productividad serán las variables claves que determinarán la permanencia de las empresas en los mercados. Y es aquí donde la logística juega un papel crucial, a partir del manejo eficiente del flujo de bienes y servicios hacia el consumidor final.

En este orden de ideas, para Reyes (2001), el concepto con el cual se introdujo a la logística se enfocaba al manejo de todas las actividades que facilitan el movimiento de productos, la coordinación de la oferta y la demanda, la optimización de la utilidad en el tiempo y la producción, para ofrecer el producto esperado, en el lugar preciso, con la cantidad requerida, en el tiempo justo a un costo adecuado.

De esta forma, todas aquellas actividades que involucran el movimiento de materias primas, materiales y otros insumos forman parte de los procesos logísticos, al igual que todas aquellas tareas que ofrecen un soporte adecuado para la transformación de dichos elementos en productos terminados: las compras, el almacenamiento, la administración de los inventarios, el mantenimiento de las instalaciones y maquinarias, la seguridad y los servicios de planta (suministros de agua, gas, electricidad, combustibles, aire comprimido, vapor, entre otros).

Desde estas consideraciones, Carranza (2005: 361), define la logística como:

Aquella parte del proceso de la cadena de valor que planifica, implementa y controla el eficiente, efectivo flujo, almacenamiento de mercaderías, servicios e información relacionada, desde el punto de origen hasta el punto de consumo para responder a los requerimientos de los clientes.

De manera que, las actividades logísticas deben coordinarse entre sí para lograr mayor eficiencia en todo el sistema productivo. Por dicha razón, la logística no debe verse como una función aislada, sino como un proceso global de generación de valor para el cliente, esto es, un proceso integrado de tareas que ofrezca una mayor velocidad de respuesta al mercado, con costos mínimos.

En ese sentido, un sistema de gestión logística se debe desarrollar a fin de asegurar que los procesos de la organización se planifiquen y se desarrollen incluyendo los requisitos logísticos del cliente, como lo manifiestan Casanovas y Cuatrecasas (2010), es necesario introducir la flexibilidad necesaria en la distribución para satisfacer las necesidades del mercado cambiante, reaccionar rápidamente ante la solicitud de órdenes de trabajo del cliente, eliminar todos los inventarios innecesarios haciendo que en los pedidos aceleren el proceso productivo.

Todos los aspectos mencionados son de vital importancia; y al ser evaluados en el contexto de la gerencia de operaciones de mantenimiento a taladros en la industria petrolera División Occidente, se observa según las entrevistas diagnósticas no estructuradas, la observación directa por parte del investigador, así como la revisión de algunas estadísticas que se llevan en estas divisiones que, no existe un almacén de materiales en cada distrito, que despache diariamente los requerimientos necesarios para el proceso productivo de manera oportuna. Encontrándose el almacén principal en el área de Tía Juana por lo que 
existe un tiempo de viaje para la entrega de los requerimientos que en ocasiones dificulta dar respuesta de manera inmediata al proceso productivo.

Destacándose, además, las compras que se deben realizar son para trabajos de inmediato, con lo que se requiere una buena estrategia para colocar el requerimiento en el sitio y hora indicada. Todo esto evidentemente se contrapone a cualquier optimización de costos e incrementa la probabilidad de accidentes, debido a que en la urgencia operativa quizás no se pidan certificados, no se hagan los controles de calidad o bien no se siga la trazabilidad de un material.

\section{Gestión logística}

Lozano (2010), considera que la gestión logística es un conjunto de actividades y procedimientos prácticos, que tienen como finalidad que todos los recursos necesarios para conseguir un fin estén disponibles en el momento, lugar, modo y cantidad precisa al mínimo coste. Visto así, será un sistema actividades interdependientes que pueden variar de una organización a otra.

Seguidamente, citando a Pires y Carretero (2007), la gestión logística es la planificación, implementación, control del flujo de efectivo, el stock de bienes y servicios e informaciones pertinentes desde el punto de origen hasta el punto de consumo, con el objetivo de atender las necesidades de los clientes.

Por su parte, Ballou (2004) define la gestión logística como un sistema que comprende determinadas funciones ejecutadas por los niveles gerenciales de la organización, para asignar los recursos, coordinar el trabajo y los rendimientos de otros empleados (ajenos a la función de gestión), con la finalidad de producir bienes $y / 0$ servicios que satisfagan las necesidades de sus clientes, de forma que se alcancen los objetivos de la empresa con altos niveles de productividad, lo que implica eficacia y eficiencia.

De acuerdo a las consideraciones que anteceden, se entiende que la gestión logística es considerada como una función operativa que comprende todas las actividades y procesos necesarios para la administración estratégica del flujo y almacenamiento de materias primas y componentes, existencias en proceso $y$ productos terminados; de tal manera, que éstos estén en la cantidad adecuada, en el lugar correcto y en el momento apropiado.

\section{Actividades de apoyo de la gestión logística}

Las actividades de apoyo dentro de las organizaciones son las responsables de proveer de todos los recursos necesarios en cuanto a personas, maquinaria y materia prima, para poder generar el valor añadido deseado por los clientes de contabilidad, compras, nominas, sistemas de información, entre otros. Benavides (2011), las define como aquellas relacionadas con la infraestructura, desarrollo del capital humano, desarrollo tecnológico, adquisición, sistemas de comunicación e información, entre otros.

Ahora bien, en los procesos logísticos, según Serra (2005), las actividades de apoyo o de soporte son aquellas que no están necesariamente ligadas al flujo de material, pero resultan claves para el funcionamiento satisfactorio de las actividades fundamentales. Este autor establece como actividades de apoyo: almacenamiento, manejo de materiales, compras, cooperaciones con producción y el mantenimiento de información. Así mismo, para 
Cuervo y Osorio (2006), en los procesos logísticos las actividades de apoyo son el conjunto de actividades que se realizan para mantener en debida forma las instalaciones físicas y tecnológicas de la empresa en donde se llevan a cabo las demás actividades. Con frecuencia, el apoyo que proporciona para sus productos o servicios es tan importante para los clientes como la calidad del producto o servicio.

En este sentido, según Anaya (2014), estas actividades apoyan a las fundamentales o clave para que estas se cumplan, y generan los recursos que precisan las demás actividades. Ejemplo: almacenamiento, manejo de materiales, compras, cooperaciones con producción y el mantenimiento de información, entre otras. De manera similar, Fontalvo y Vergara (2010) las definen como actividades de soporte del sistema logístico, se encuentran en las interfaces entre la logística, la producción y la comercialización, se incluyen: almacenamiento, manejo de mercancías, compra, planificación del producto, empaque, gestión de información.

A efectos del investigador, las actividades de apoyo son aquellas que dan apoyo a las actividades fundamentales del proceso logístico, aportándoles los recursos necesarios. Son actividades en las que el cliente es interno, se consumen dentro de los procesos logísticos por las mismas personas de la organización. También podrían calificarse como actividades de soporte a las fundamentales para que estas se cumplan, y les garantizan los recursos que precisan las demás actividades. Cabe resaltar que para su estudio se clasificarán de acuerdo a los criterios de Serra (2005) y Anaya (2014), por lo cual se asumirán como indicadores de esta dimensión: almacenamiento, manejo de materiales, compras, cooperaciones con producción y el mantenimiento de información, descritas a continuación:

\section{Almacenamiento}

Para Serra (2005) el almacenaje agrupa las decisiones asociadas a la determinación del espacio requerido, el diseño, configuración de los almacenes y la disposición de los productos en su interior, añadiendo valor al producto en el tiempo, asociado a la custodia y salvaguarda de sus condiciones. De acuerdo a Ballou (2004), es la actividad de soporte a la logística que implica el resguardo de los diferentes tipos de mercancía; manejado a través de una política de inventario, controla físicamente y mantiene todos los artículos inventariados. Al elaborar la estrategia de almacenaje se deben definir de forma coordinada el sistema de gestión del almacén y el modelo de almacenamiento.

Silva (2006), lo define como una actividad de gestión de almacenamiento o almacenaje, un sistema que determina los criterios para seleccionar el material que ha de salir del almacén para atender una petición concreta.

Ahora bien, reflexionando sobre lo expuesto, para el investigador, la actividad de almacenaje brinda apoyo al proceso logístico, permitiendo el custodio de los insumos, materiales o productos semi-elaborados que se requieran para el proceso de transformación productivo, así como para el resguardo de los productos finales. Por ello, la eficiente gestión de la logística dependerá en alto grado de un correcto proceso de almacenaje, que garantice las condiciones requeridas para el mantenimiento de estos materiales y productos en condiciones óptimas para su procesamiento o venta. 


\section{Manejo de materiales}

Según Serra (2005) al manejar las mercancías se realizan una serie de actividades que implican desde la selección adecuada del equipo de manipulación de materiales en el almacén, hasta los detalles de preparación de los pedidos y devolución de productos defectuosos.

Agrega Ballou (2004) que existen aspectos muy importantes del manejo de materiales, además de geometría y herramientas, incluye movimiento de hombres, máquinas, tecnologías e información. El sistema de flujo, o tratamiento de mercancías debe de apoyar los objetivos de la recepción, selección, inspección, inventario, contabilidad, empaque, ensamble y otras funciones de producción.

Ahora bien, para Carrasco (2005), está actividad está ligada al almacenamiento y el inventario. La misma es una actividad de mano de obra intensa, ya que la mayor parte del manejo de materiales se realiza de manera manual, o en el mejor de los casos en forma semiautomática. La disposición de la mercancía, la magnitud con que se utiliza el equipo, y el grado de automatización afectan a los costos del manejo de materiales.

Para efecto de esta investigación, se debe considerar que esta actividad se relaciona con la gestión logística en cuanto a las funciones primordiales que desarrolla como la selección del equipo, procedimientos de preparación de pedidos, almacenamiento y recuperación de mercancías; siendo eje medular para las empresas bajo estudio, dado que es una de las actividades con mayor frecuencia de utilización.

\section{Compras}

Las compras, según Serra (2005), es una actividad de apoyo al proceso logístico que tiene como objetivo adquirir aquellos bienes 0 servicios que la empresa necesita, garantizando el abastecimiento de las cantidades requeridas en el momento preciso y en las mejores condiciones posibles de calidad y precio. A través de ella se seleccionan las fuentes, se determinan las cantidades a adquirir y el momento de efectuar las adquisiciones y la planificación de los productos. Impacta en la gestión de inventario.

En esta perspectiva, Mercado (2006) define la compra como aquella operación que involucra todo el proceso de ubicación de proveedor o fuentes de abastecimiento, adquisición de materiales a través de negociaciones de precio y condiciones de pago con el proveedor elegido y la recepción de las mercaderías correspondientes para controlar y garantizar el suministro de la adquisición.

En este orden de ideas, para Butter y Linse (2008), la compra para las compañías se compone de seis pasos claros. En el primer paso la compañía identifica una necesidad, para la cual la respuesta es la compra de un producto, y el paso final es la ejecución de un contrato. Los pasos intermedios construyen un proceso organizado e informado que tiene como resultado que la compañía compre el producto correcto para cubrir su necesidad de un proveedor calificado cuyo producto es el más duradero por el precio.

Afirman los autores citados que el proceso puede variar de una empresa a otra debido a la actividad principal. La empresa industrial centra sus compras en los materiales que utiliza para elaborar el producto. La empresa comercial, por su parte, busca conseguir el producto a un precio competitivo. $Y$, por último, para la empresa de servicios la variable principal es satisfacer las necesidades del cliente. Algunas 
veces el proceso de compras es largo y complejo debido a que para tomar una decisión final se necesita información o respuesta de otras empresas.

Ahora bien, de acuerdo a Moore (2006), aunque esta actividad pueda constituir de vital importancia para la empresa, como puede verse, solo es una parte de todas las operaciones que debe realizar para aprovisionarse. La eficacia de la gestión de compras se medirá en función de: el control de gastos y costos, el manejo de stocks mínimos, la habilidad para encontrar fuentes alternativas de abastecimiento, y su posibilidad de investigar y conocer nuevos materiales disponibles en el mercado.

De lo expuesto, el investigador infiere que las compras, como actividad de apoyo a la logística, involucra la adquisición de materias primas, componentes y suministros para la organización. Las actividades asociadas a esta parte del proceso logístico incluyen desde la detención de la necesidad, hasta la especificación de la forma en la que se recibirán los bienes o servicios.

\section{Cooperaciones con producción}

Se utiliza para especificar cantidades adicionales, secuencia y rendimiento del tiempo de producción, programación de suministro para la producción y operaciones. Para Jiménez y Hernández (2002), son relaciones de largo plazo, coordinadas con equipo multiempresa, con previsiones compartidas, planificación conjunta y otras características relevantes como el compartir riesgos y ganancias.

Según Silva (2006), este tipo de relaciones también son conocidas como relaciones de sociedad entre clientes y proveedores. Como se puede intuir, las relaciones de colaboración, aparecen como un tipo de relación que permite a las empresas beneficiarse de las ventajas de ambos enfoques extremos y minimizar los problemas asociados a las relaciones distantes.

Desde la perspectiva de la logística, los beneficios obtenidos a través de la colaboración permiten a las empresas gestionar el proceso de satisfacción de los pedidos de compra de manera eficaz y eficiente cumplir con los requerimientos de los clientes con mayor celeridad y enfrentar unidas las fluctuaciones de la demanda mediante la introducción de cambios más rápidos en la planificación de los recursos destinados a la producción.

A objeto de esta investigación, la falta de una coordinación adecuada de las relaciones entre proveedor y cliente sin lugar a dudas trae consigo múltiples problemas (por ejemplo, fallos en la recepción, incumplimiento de las condiciones de los pedidos, fallas en la fecha y hora de entrega, problemas de descarga, rechazo de mercancías, errores de facturación, otros), que afectan al precio de los productos, en consecuencia, a la competitividad de las empresas.

\section{Mantenimiento de información}

Ballou (2004) explica que el mantenimiento de información es una actividad donde se desenvuelve la recopilación, almacenamiento y manipulación de información, análisis de datos y procesos de control. De igual manera para Silva (2006) el mantenimiento de información apoya a todas las actividades de la logística, ya que suministra la información necesaria para la planeación y el control. Se podrían resumir los elementos que intervienen en el proceso de la logística, aclarando que muchas de estas actividades se repiten a lo largo de este proceso en parte de la cadena de suministro de una empresa es que se presentan. 
En este contexto para el investigador, el mantenimiento de la información da soporte a todo el sistema logístico, haciendo énfasis en la planificación y control de necesidades.

\section{MÉTODO}

Metodológicamente se tipificó como una investigación descriptiva, con un diseño de campo no experimental y transeccional. La población quedó constituida por las gerencias de operaciones y mantenimiento a taladro en la industria petrolera división Occidente, específicamente: División de operaciones y mantenimiento a taladro Sur del Lago de Trujillo, Costa Oriental del Lago y Costa Occidental., siendo las unidades informantes: 3 gerentes y 15 líderes con experiencia en gestión logística.
La recolección de la información se realizó a través de un cuestionario con alternativas de frecuencia de cinco opciones de respuestas compuesto por 11 ítems, el cual fue validado previamente por 5 expertos. Para determinar la confiabilidad del instrumento se realizó una prueba piloto a 6 sujetos con características similares a la población seleccionada y luego se calculó la confiabilidad mediante el método de Alfa de Cronbach, cuyo valor fue de 0,98 ubicándose con un nivel de muy alta magnitud según el baremo establecido.

La información fue procesada a través de la estadística descriptiva específicamente mediante la media aritmética. Para tal fin, el investigador diseño un baremo de interpretación, tal como se aprecia en el cuadro 1.

Cuadro 1. Baremo para la interpretación del promedio

\begin{tabular}{cccc}
\hline ALTERNATIVA & INTERVALO & CATEGORÍA & INTERPRETACIÓN \\
\hline Siempre & $4.21 \leq 5.00$ & Muy alta presencia & Fortaleza para la gestión analizada \\
Casi siempre & $3.41<4.20$ & Alta presencia & \\
A veces & $2.61<3.40$ & Moderada presencia & \\
Casi nunca & $1.81<2.60$ & Baja presencia & Debilidad para la gestión analizada \\
Nunca & $1.00 \leq 1.80$ & Muy baja presencia & \\
\hline
\end{tabular}

Fuente: El autor (2019)

\section{RESULTADOS}

Como se puede apreciar en la tabla 1 , se presenta el resumen para la dimensión actividades de apoyo, perteneciente a la gestión logística en las gerencias de operaciones y mantenimiento a taladro en la industria petrolera división occidente, el cual muestra un valor de 3,51, reflejando de acuerdo a la opinión de los encuestados alta presencia de aquellas actividades, que si bien, no son parte de la actividad principal de la organización, son necesarias e imprescindibles para la buena gestión y desarrollo de la misma, representando en tal sentido una fortaleza para las gerencias bajo estudio. 
Tabla 1: Dimensión: Actividades de apoyo

\begin{tabular}{ccc}
\hline INDICADORES & PROMEDIO & CATEGORÍA \\
\hline Almacenamiento & 3,47 & Alta presencia/Fortaleza \\
Manejo de materiales & 3,45 & Alta presencia/Fortaleza \\
Compras & 3,49 & Alta presencia/Fortaleza \\
Cooperación con producción & 3,63 & Alta presencia/Fortaleza \\
Mantenimiento de información & 3,50 & Alta presencia/Fortaleza \\
\hline PROMEDIO DIMENSIÓN & $\mathbf{3 , 5 1}$ & ALTA PRESENCIA/FORTALEZA
\end{tabular}

Fuente: El autor (2019)

Al analizar los promedios totales alcanzados para la dimensión en cada uno de sus indicadores se percibe que, todas las actividades de apoyo analizadas poseen alta presencia en la gestión logística que desarrollan, con concentraciones de respuestas afirmativas que así lo demuestran, destacándose la cooperación de producción $(3,63)$, mantenimiento de información $(3,50)$ y compras $(3,49)$; seguidos almacenamientos $(3,47)$ y el manejo de materiales $(3,45)$, en este orden.

Lo anterior mostrado, permite verificar alta coincidencia con Serra (2005), para quien las actividades de apoyo o de soporte son aquellas que no están necesariamente ligadas al flujo de material, pero resultan claves para el funcionamiento satisfactorio de las actividades fundamentales, tales como: almacenamiento, manejo de materiales, compras, cooperación con producción y mantenimiento de información. De igual manera se valida lo postulado por Anaya (2014), cuando afirma que, estas actividades apoyan a las fundamentales o claves para que estas se cumplan, y generan los recursos que precisan las demás actividades, y cita como ejemplos: almacenamiento, manejo de materiales, compras, cooperación con producción y mantenimiento de información, entre otras.

En esta perspectiva, a juicio del investigador, las gerencias bajo estudio están soportando su gestión logística en actividades encaminadas al logro de los objetivos, al mostrar alta presencia en las actividades de apoyo aportándole los recursos necesarios a las actividades fundamentales de su proceso logístico.

Ahora bien, al detalle de cada uno de los indicadores utilizados para medir la dimensión a continuación se especifican los resultados obtenidos al respecto, mostrados en la tabla 2 , donde se recoge el resultado de cada uno de sus indicadores. De esta forma, se evidencia alta presencia para cada uno de los indicadores, otorgándole fortaleza a la gestión logística.

En relación al indicador almacenamiento, se evidencia alta presencia de espacios acorde para el almacenamiento de los insumos necesarios para las operaciones de mantenimiento, y del cumplimiento de normativas para el almacenamiento de los insumos necesarios, atrojándole fortaleza a la gestión logística.

Esta situación, de alta presencia del indicador, valida la posición teórica planteada por Ballou (2004), cuando afirma que el almacenaje es la actividad de soporte a la logística que implica el resguardo de los diferentes tipos de mercancía; manejado a través de la política de inventario, controla físicamente y mantiene todos los artículos inventariados. 
Tabla 2. Indicadores de la dimensión actividades de apoyo

\begin{tabular}{|c|c|c|c|c|}
\hline INDICADOR & $\mathbf{N}^{\circ}$ & ITEMS & $\overline{\bar{X}}$ & CATEGORIA \\
\hline \multirow{3}{*}{ 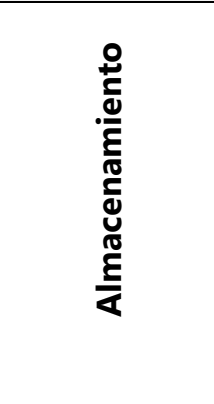 } & 1 & $\begin{array}{l}\text { Se cuenta con espacios acorde para el } \\
\text { almacenamiento de los insumos necesarios para las } \\
\text { operaciones de mantenimiento }\end{array}$ & 3,48 & $\begin{array}{l}\text { Alta presencia/ } \\
\text { Fortaleza }\end{array}$ \\
\hline & 2 & $\begin{array}{l}\text { Se cumple con normativas para el almacenamiento } \\
\text { de los insumos necesarios para las operaciones de } \\
\text { mantenimiento. }\end{array}$ & 3,46 & $\begin{array}{l}\text { Alta presencia/ } \\
\text { Fortaleza }\end{array}$ \\
\hline & & INDICADOR & 3,47 & $\begin{array}{l}\text { Alta presencia/ } \\
\text { Fortaleza }\end{array}$ \\
\hline \multirow{4}{*}{ 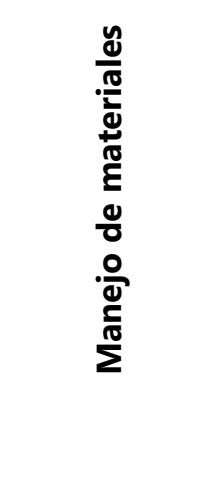 } & 3 & $\begin{array}{l}\text { La gerencia de operaciones de mantenimiento de } \\
\text { taladros cuenta con equipos seguros para el } \\
\text { traslado de los productos adquiridos }\end{array}$ & 3,44 & $\begin{array}{l}\text { Alta presencia/ } \\
\text { Fortaleza }\end{array}$ \\
\hline & 4 & $\begin{array}{l}\text { El personal de la unidad está capacitado para el } \\
\text { manejo de productos adquiridos en grandes } \\
\text { cantidades }\end{array}$ & 3,46 & $\begin{array}{l}\text { Alta presencia/ } \\
\text { Fortaleza }\end{array}$ \\
\hline & & INDICADOR & 3,45 & $\begin{array}{l}\text { Alta presencia/ } \\
\text { Fortaleza }\end{array}$ \\
\hline & 5 & $\begin{array}{l}\text { Se realiza análisis de las necesidades para realizar } \\
\text { las operaciones de mantenimiento }\end{array}$ & 3,51 & $\begin{array}{l}\text { Alta presencia/ } \\
\text { Fortaleza }\end{array}$ \\
\hline \multirow{3}{*}{$\frac{\mathscr{y}}{\frac{0}{0}}$} & 6 & $\begin{array}{l}\text { Se cuenta con un registro especificado de las } \\
\text { operaciones de mantenimiento }\end{array}$ & 3,53 & $\begin{array}{l}\text { Alta presencia/ } \\
\text { Fortaleza }\end{array}$ \\
\hline & 7 & $\begin{array}{l}\text { Se realizan las compras necesarias para las } \\
\text { operaciones de mantenimiento en las cantidades } \\
\text { necesarias y económica con la calidad adecuada }\end{array}$ & 3,42 & $\begin{array}{l}\text { Alta presencia/ } \\
\text { Fortaleza }\end{array}$ \\
\hline & & INDICADOR & 3,49 & $\begin{array}{l}\text { Alta presencia/ } \\
\text { Fortaleza }\end{array}$ \\
\hline \multirow{3}{*}{ 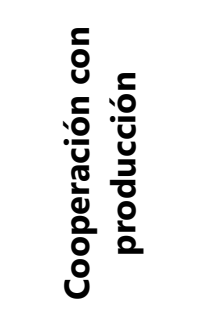 } & 8 & $\begin{array}{l}\text { Las operaciones de mantenimiento de taladros se } \\
\text { coordinan entre todas las gerencias }\end{array}$ & 3,68 & $\begin{array}{l}\text { Alta presencia/ } \\
\text { Fortaleza }\end{array}$ \\
\hline & 9 & $\begin{array}{l}\text { Las operaciones de mantenimiento de taladros se } \\
\text { cumplen según los requerimientos de las gerencias }\end{array}$ & 3,58 & $\begin{array}{l}\text { Alta presencia/ } \\
\text { Fortaleza }\end{array}$ \\
\hline & & INDICADOR & 3,63 & $\begin{array}{l}\text { Alta presencia/ } \\
\text { Fortaleza }\end{array}$ \\
\hline \multirow{4}{*}{ 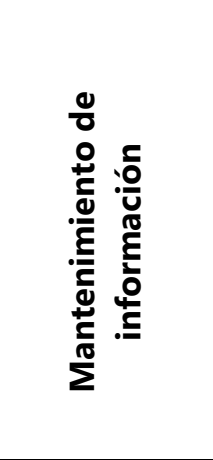 } & 10 & $\begin{array}{l}\text { Las gerencias suministran la información necesaria } \\
\text { para elaborar el plan de operaciones de } \\
\text { mantenimiento }\end{array}$ & 3,51 & $\begin{array}{l}\text { Alta presencia/ } \\
\text { Fortaleza }\end{array}$ \\
\hline & 11 & $\begin{array}{l}\text { Las gerencias cuentan con un sistema de } \\
\text { información sobre el inventario de productos } \\
\text { requeridos para las operaciones de mantenimiento }\end{array}$ & 3,49 & $\begin{array}{l}\text { Alta presencia/ } \\
\text { Fortaleza }\end{array}$ \\
\hline & & INDICADOR & 3,50 & $\begin{array}{l}\text { Alta presencia/ } \\
\text { Fortaleza }\end{array}$ \\
\hline & & DIMENSIÓN & 3,51 & $\begin{array}{c}\text { Alta presencia/ } \\
\text { Fortaleza }\end{array}$ \\
\hline
\end{tabular}

Fuente: El autor (2019) 
En este punto, a criterio del investigador, el almacenaje en las gerencias analizadas, alcanzan la función para la cual se utiliza esta actividad de soporte en la gestión logística, dado que se muestra con alta presencia, garantizando las condiciones requeridas para el mantenimiento de los materiales y productos en condiciones óptimas para su procesamiento o venta.

Con relación al indicador manejo de materiales, los hallazgos demuestran fortalezas para la gestión logística, al ubicarse en alta presencia el contar con equipos seguros para el traslado de los productos adquiridos y de personal capacitado para el manejo de productos adquiridos en grandes cantidades.

Estos señalamientos validan, en gran medida, lo planteado por Serra (2005), para quien al manejar las mercancías se realizan una serie de actividades que implican desde la selección adecuada del equipo de manipulación de materiales en el almacén, hasta los detalles de preparación de los pedidos y devolución de productos defectuosos.

Visto así, para el investigador, los resultados manifiestan que el tratamiento de mercancías, como parte de las actividades de apoyo a la gestión logística que ejecutan las gerencias bajo estudio, considerándola en relación directa con la gestión logística en cuanto a las funciones primordiales que ésta desarrolla como: selección del equipo, procedimientos de preparación de pedidos, almacenamiento y recuperación de mercancías; siendo eje medular para las empresas bajo estudio, dado que es una de las actividades con mayor nivel de presencia.

Seguidamente, se aprecia alta presencia del indicador compras, al considerar los encuestados que se realizan análisis de las necesidades para realizar las operaciones de mantenimiento, asimismo cuenta con un registro especificado de las operaciones de mantenimiento y realizan las compras necesarias para las operaciones de mantenimiento en las cantidades necesarias y económica con la calidad adecuada.

Según los resultados, las compras en la gestión logística bajo análisis, muestran alta presencia, lo cual coincide con Serra (2005), para quien las compras son una actividad de apoyo al proceso logístico, que tiene como objetivo adquirir aquellos bienes o servicios que la empresa necesita, garantizando el abastecimiento de las cantidades requeridas en el momento preciso $y$ en las mejores condiciones de calidad y precio.

En virtud de lo expuesto, a criterio del investigador, en las gerencias bajo estudio, se logra procesar las compras como una actividad de apoyo a la logística, involucrando la adquisición de materias primas, componentes y suministros para la organización. Las actividades asociadas a las compras incluyen desde la detención de la necesidad, hasta la especificación de la forma en la que se recibirán los bienes o servicios.

En este recorrido de ideas, se presentan los resultados del indicador cooperación con producción, el cual representa una fortaleza para la gestión logística, por cuanto con alta presencia las operaciones de mantenimiento a taladros se coordinan entre todas las gerencias y se cumplen según los requerimientos de las gerencias.

De tal forma, se valida de forma alta los postulados de Silva (2006), para quien, este tipo de relaciones son conocidas como relaciones de sociedad entre clientes $y$ 
proveedores y como se puede intuir, aparecen como un tipo de relación que permite a las empresas beneficiarse de las ventajas de ambos enfoques extremos y minimizar los problemas asociados a las relaciones distantes.

Para el investigador, los resultados ponen de manifiesto que, los beneficios obtenidos a través de la colaboración permiten a las gerencias bajo estudio gestionar el proceso de satisfacción de los pedidos de compra de manera eficaz y eficiente cumplir con los requerimientos de los clientes con mayor celeridad y enfrentar unidas las fluctuaciones de la demanda mediante la introducción de cambios más rápidos en la planificación de los recursos destinados a la producción.

Finalmente, se presentan los resultados del indicador mantenimiento de información, ubicándolo en la categoría de alta presencia, al referir los encuestados que las gerencias suministran la información necesaria para elaborar el plan de operaciones de mantenimiento y cuentan con un sistema de información sobre el inventario de productos requeridos para las operaciones de mantenimiento.

Esta situación, de alta presencia del indicador, valida la posición teórica planteada por Silva (2006), cuando afirma que el mantenimiento de información apoya a todas las actividades de la logística, ya que suministra la información necesaria para la planeación y el control. En este punto, a criterio del investigador, el mantenimiento de información, facilita el soporte a todo el sistema logístico, haciendo énfasis en la planificación y control de necesidades.

Para cerrar, el investigador observa que en las gerencias analizadas se da con alta presencia las actividades propias de la gestión logística, desde la óptica de los autores que fundamentan la teoría de esta investigación, asumiéndolas como el flujo de materiales e información tanto interna con externa a la organización, por lo tanto, su logística es el enlace entre los mercados y la operación de las mismas.

\section{CONCLUSIONES}

Se caracterizó las actividades de apoyo de la gestión logística en la industria petrolera división occidente, evidenciándose que hay alta presencia de un conjunto de actividades que sustentan a las actividades primarias y se apoyan entre sí, proporcionando insumos comprados, tecnología, recursos humanos, además de varias funciones de todas las gerencias de operaciones y mantenimiento a taladro.

En este sentido, la gestión logística engloba todas las áreas de la industria petrolera, implicando la realización de diferentes tipos de actividades de apoyo, desde la programación de compras hasta satisfacer las necesidades del cliente y sus expectativas, al menor costo posible.

\section{REFERENCIAS}

Anaya, J. (2014). El diagnóstico logístico: Una metodología para promover mejoras competitivas. Libros profesionales de empresa. Libros profesionales de empresa. Editorial ESIC. Madrid. España

Ballou, R. (2004). Logística. Administración de la cadena de suministro. Quinta Edición. Editorial Pearson Educación. México

Benavides, R. (2011). Administración logística. Editorial McGraw-Hill. México

Butter, F.y Linse, K. (2008). Rethinking procurement in the era of globalization.MIT Sloan Management Review. Pp. 76-80 
Carranza, O. (2005). Logística: Mejores prácticas en Latinoamérica. Publicado por International Thomson. México

Carrasco, J. (2005). Evolución de los enfoques y conceptos de la logística. Editado por la Universidad Politécnica de Madrid. España

Casanovas, A. y Cuatrecasas, LI. (2010). Logística empresarial. Sexta edición. Ediciones Gestión 2000 S.A. Barcelona. España

Cuervo, J. y Osorio, J. (2006). Costeos basados en actividades $A B C$ : gestión basada en Actividades. Tercera Edición. Deusto ediciones. Madrid. España

Jiménez, J y Hernández, S (2002). Marco conceptual de la cadena de suministro: un nuevo enfoque logístico. Coordinación operativa del instituto mexicano del transporte.Santanilla. Disponible en: http://www.azc.uam.mx/alumnos/tradeoff/d ocu/marco.pdf

Lozano, J. (2010). Cómo y dónde optimizar los costes logísticos: en el sistema integral de operaciones y en las diferentes áreas de actividad logística. Segunda edición. FC Editorial. España

Mercado, S. (2006). Compras: principios y aplicaciones. Cuarta Edición. Editorial Limusa, S.A. México

Moore, C. (2006). Administración de pequeñas empresas: Enfoque emprendedor. $13^{\mathrm{a}}$ Edición. Editorial Thomson. España

Pires, S., y Carretero, L. (2007). Gestión de la cadena de suministros. Editorial Mc Graw Hill. España

Reyes, A (2001). Logística: Optimización de Tiempo y Productividad. Revista Ejecutivos de Finanzas. Abril, No. 04 México

Serra, D. (2005). Logística empresarial en el nuevo milenio. Editorial Gestión 2000. Barcelona. España

Silva, F. (2006). Principios y estrategias de la gestión por procesos. Primera edición. Editorial Universitas. Argentina 